\title{
Implication of Microsatellite Instability Pathway in Outcome of Colon Cancer in Moroccan Population
}

\author{
Fatima El Agy $\mathbb{D}^{1},{ }^{1}$ Ihssane El Otmani $\mathbb{D}^{1},{ }^{1}$ Asmae Mazti, ${ }^{2}$ Nada Lahmidani, ${ }^{3}$ \\ Abdelmalek Oussaden, ${ }^{4}$ Mohamed El Abkari, ${ }^{3}$ El Bachir Benjelloun, ${ }^{4}$ Wadih Moukit $\left(\mathbb{D},{ }^{5}\right.$ \\ Hicham El Bouhaddouti, ${ }^{4}$ Imane Toughrai, ${ }^{4}$ Karim Ibn Majdoub Hassani, ${ }^{4}$ Khalid Maazaz, ${ }^{4}$ \\ Zineb Benbrahim (D), ${ }^{6}$ Nawfal Mellas, ${ }^{6}$ Karima El Rhazi, ${ }^{7}$ Karim Ouldim, ${ }^{8}$ Sanae El Bardai, ${ }^{2}$ \\ Sidhi Adil Ibrahimi, ${ }^{9}$ Khalid Ait Taleb, ${ }^{9}$ Sanae Bennis, ${ }^{10}$ and Chbani Laila ${ }^{2}$
}

${ }^{1}$ Medical Center of Biomedical and Translational Research, Hassan II University Hospital, Fez, Morocco

${ }^{2}$ Department of Pathological Anatomy, Hassan II University Hospital, Fez, Morocco

${ }^{3}$ Department of Gastroenterology, Hassan II University Hospital, Fez, Morocco

${ }^{4}$ Department of Visceral Surgery, Hassan II University Hospital, Fez, Morocco

${ }^{5}$ Department of Obstetrics and Gynecology, Military Training Hospital Mohammed V, Rabat, Morocco

${ }^{6}$ Department of Oncology, Hassan II University Hospital, Fez, Morocco

${ }^{7}$ Laboratory of Epidemiology, Faculty of Medicine and Pharmacy, Fez, Morocco

${ }^{8}$ Department of Medical Genetics and Ontogenetic, Hassan II University Hospital, Fez, Morocco

${ }^{9}$ Department of General Surgery, Hassan II University Hospital, Fez, Morocco

${ }^{10}$ Biomedical and Translational Research Laboratory, Faculty of Medicine and Pharmacy, Morocco

Correspondence should be addressed to Fatima El Agy; fatima.elagy@usmba.ac.ma

Received 8 May 2019; Revised 16 August 2019; Accepted 13 September 2019; Published 7 December 2019

Academic Editor: Stamatios E. Theocharis

Copyright ( 2019 Fatima El Agy et al. This is an open access article distributed under the Creative Commons Attribution License, which permits unrestricted use, distribution, and reproduction in any medium, provided the original work is properly cited.

\begin{abstract}
Background. Tumors with microsatellite instability (MSI tumors) have distinct clinicopathological features. However, the relation between these tumor subtypes and survival in colon cancer remains controversial. The aim of this study was to evaluate the overall survival (OS) in patients with MSI phenotype, in FES population. Methods. The expression of MMR proteins was evaluated by immunohistochemistry for 330 patients. BRAF, KRAS, and NRAS mutations were examined by Sanger sequencing and pyrosequencing methods. The association of MSI status with a patient's survival was assessed by the Kaplan-Meier method and log-rank test. Results. The mean age was 54.6 years (range of $19-90$ years). The MSI status was found in $11.2 \%$ of our population. MSI tumors were significantly associated with male gender, younger patients, stage I-II, right localization, and a lower rate of lymph node and distant metastasis. The OS tends to be longer in MSI tumors than MSS tumors (109.71 versus 74.08), with a difference close to significance $(P=0.05)$. Conclusion. Our study demonstrates that MSI tumors have a particular clinicopathological features. The results of survival analysis indicate that the MSI status was not predictive of improved overall survival in our context with a lower statistical significance $(P=0.05)$ after multivariate analysis.
\end{abstract}

\section{Introduction}

Microsatellite instability (MSI) or mismatch repair deficient (dMMR) is one of the main pathogenetic pathways leading to the development of colorectal cancer [1]. MSI phenotype is due to dysfunction of a DNA mismatch repair (MMR) system in microsatellite replication [2]. The MMR system is composed of four MMR genes and their encoded proteins (MLH1, MSH2, MSH6, and PMS2) [3]. These proteins form heterodimers that repair DNA damage (mismatches as well as short insertion or deletion loops) [4]. In hereditary nonpolyposis CCRs (e.g., Lynch syndrome), 90\% of the MMR alterations are mainly due to constitutional mutations of the MLH1 and MSH2 gene (more rarely MSH6 or PMS2 gene) 
or alteration in EPCAM (TACSTD1) gene that causes epigenetic silencing of MSH2 $[5,6]$, while 10 to $15 \%$ of all sporadic CCRs are due to hypermethylation of CpG islands in the MLH1 promoter [6].

Several studies have shown that dMMR tumors have special clinicopathological features, including poor differentiation, right colon location, abundant tumor-infiltrating lymphocytes, and mucinous histology [7]. Furthermore, these tumors are associated with the presence of BRAF mutations [8]. MMR tumors are diagnosed in $15-20 \%$ of localized CCRs, especially in stage II, although they represent $3-5 \%$ of metastatic CCRs [9].

Variable results are reported in the literature, about the association between MSI status and survival in colorectal cancer. Tumors diagnosed at stage II or III with MSI have better prognosis than MSS tumors [10, 11]. In addition, many studies have demonstrated that CCRs with MSI status showed poorer response to 5-fluorouracil (5FU) compared to CCRs with MSS status [10]. For patients with stage III and MSI, it has been confirmed that only those with suspected germinal mutations can benefit from treatment with $5 \mathrm{FU}[11]$.

In the Moroccan population, few studies have reported the prognostic factors of MSI colon cancer. Therefore, in this study, we aimed to assess the frequency of loss in MMR protein expression, to compare the clinical and pathological features of MSI versus MSS colon cancers, and to evaluate the survival rates in patients with MSI tumors in association with other clinicopathological features, for the first time in the FES population.

\section{Materials and Methods}

2.1. Ethics. This study protocol was reviewed and approved by Hassan II University Hospital Ethics Committee of FEZ, Morocco, under reference no. 13/18. All patients gave informed consent before the start of the study.

A total of 330 patients diagnosed with colon cancer were included in this study, in the Department of Pathology of Hassan II University Hospital, Fez, Morocco, from 2013 to 2019. Medical charts have been reviewed, and patients have been selected using the following selection criteria: (a) patients had histologically confirmed primary adenocarcinoma (b) all cases with pathologic stage I-IV colon cancer and underwent surgical resection for CC tumor. However, patients were excluded if their records were incomplete and without histological confirmation of colon adenocarcinoma and if they had rectal cancer (Figure 1).

The clinical and pathological data including age, gender, main histological pattern, tumor grade, tumor stage, numbers of dissected regional lymph nodes, family history of colon cancer, follow-up, and outcome have been obtained from the patient's medical records and pathology reports.

2.2. Identification of HNCCP Patients. Family history and clinical data were reviewed to determine patients who fulfilled the Amsterdam (I-II) criteria and met the Bethesda guidelines for molecular Lynch syndrome (LS) testing. However, any patient did fulfill the clinical features concerning for LS.

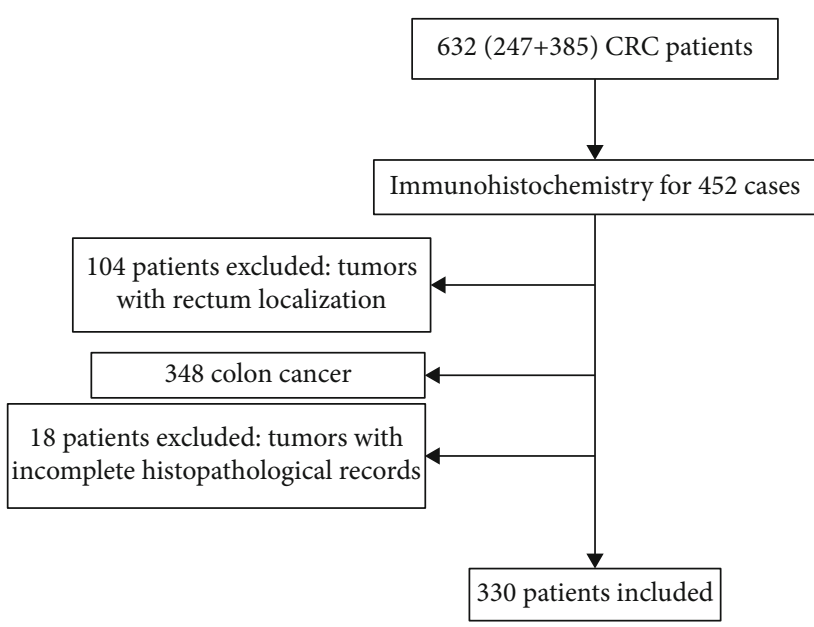

Figure 1: Flow chart of a patient's enrollment.

2.3. Detection of MMR Protein Expression by Immunohistochemistry. The mismatch repair tumor status (MSS or MSI) was assessed by immunohistochemistry (IHC) to detect the intact or the loss expression of the MMR proteins (MLH1, PMS2, MSH2, and MSH6).

The IHC study was assessed on unstained formalin-fixed paraffin-embedded (FFPE) tumor tissue sections of $5 \mu \mathrm{m}$ thickness, on the automated immunostainer Ventana Benchmark ULTRA. We have employed monoclonal antibodies specific for each MMR protein, MLH1 (G168-728/CELL MARQUE), MSH2 (G219-1129/CELL MARQUE), MSH6 (44/CELL MARQUE), and PMS2 (MRQ-28/CELL MARQUE). Adjacent normal tissue (lymphocytes or normal glandular cells) was used as an internal control for positive staining (should always show staining).

\subsection{Full RAS and BRAF Mutation Analysis}

2.4.1. Genomic DNA Extraction. Tumoral DNA was extracted from paraffin-embedded tumor sections. The blocks with higher proportion of tumors cells were selected by a pathologist on hematoxylin-, safran-, and eosin-stained slides. From the selected FFPE tumor block, $4-8$ sections of $5 \mu \mathrm{m}$ thickness were obtained for DNA extraction using the QIAamp DNA FFPE Tissue Kit (Invitrogen), according to the manufacturer's instructions.

2.4.2. BRAF Mutation Analysis. BRAF testing was performed for 200 patents, using Sanger sequencing to distinguish sporadic dMMR CC that exhibits the V600E mutation in the BRAF oncogene. DNA was amplified using PCR (Master Mix (2X) kits) according to the manufacturer's protocols. The purified PCR products were sequenced using the direct sequencing with BigDye Terminator V3.1 Cycle Sequencing Kit (ABI Prism) and analyzed on Applied Biosystems 3500Dx Genetic Analyzer (Applied Biosystems).

2.4.3. RAS Mutation Analysis. KRAS and NRAS molecular testing was performed using Sanger sequencing and pyrosequencing methods. Pyrosequencing was performed on the Qiagen PyroMark Q24 device according to the CE-IVDmarked therascreen RAS Pyro Kit Handbook. Sanger 
sequencing was performed as described previously (BRAF mutation testing).

2.5. Statistical Analysis. Statistical analysis was performed using IBM SPSS Version 20.0. The associations between the clinicopathological features of tumors and the microsatellite status (MSI/MSS) were evaluated using a chi-square test or Fisher exact tests. Tests were statistically significant when $P<0.05$. An unpaired t-test with Welch's correction was used to analyze continuous data. Survival rates were analyzed using the Kaplan-Meier method, and survival curves were compared with the log-rank test. Analysis was performed using a Cox proportional hazard model to identify prognostic factors. Factors that were significant in univariate analysis were included in the multivariate model.

\section{Results}

3.1. Patient Characteristics. The mean age was 54.62 years (range of 16-90 years). There was a slight predominance of male gender with 174 (52.7\%) men and 156 (47.3\%) women patients. The left colon was the most frequent site of CC in our population $(n=254,77 \%)$, while the right colon was diagnosed in 76 cases (23\%). Adenocarcinoma was the main histological type of CC in our study with 278 (84.2\%) cases. The moderate and the well differentiation grades were found, respectively, in 128 (38.9\%) cases and $173(52.6 \%)$ cases, despite the fact that only $28(8.5 \%)$ cases were poorly differentiated. Regarding the pathologic stage of tumors, 161 (48.8\%) were stage II, 73 (22.1\%) were stage III, 68 (20.6\%) were stage IV, and 28 were stage I (8.5\%). 68 (20.6\%) patients were classified as the metastatic group. All clinicopathological features of patients are summarized in Table 1.

Among the total of 330 colon cancer (CC) patients who prospectively tested for microsatellite instability during the study period, 293 were MSS (88.8\%) and 37 MSI (11.2\%) (Figure 2). Of the 37 MSI tumors, the loss of expression of MMR proteins was as follows: MLH1/PMS2 $(n=18)$ (5.4\%), MSH2/MSH6 $(n=11)(3.3 \%)$, MSH6 $(n=1)(0.3 \%)$, PMS2 $(n=3)(1 \%)$, and MLH1 $(n=4)(1.3 \%)$.

\subsection{BRAF, KRAS, and NRAS Analyses}

3.2.1. BRAF Analysis. BRAF V600E mutation testing was performed on all patients with MSI tumors (37 patients) and on 163 patients with MSS tumors, while we did not find any case with both $M L H 1$ protein expression loss and BRAF mutation.

3.2.2. KRAS and NRAS Analyses. RAS analysis was performed in 116 patients. We detected KRAS exon 2 mutations in 36 patients (33.3\%). Among the 80 patients with KRAS exon 2 wild-type (69.0\%), we identified two mutations in KRAS exon 4 (codon $146(1.7 \%)$ ). However, no mutation was detected in the NRAS gene (0\%) (Figure 3).

3.3. Pathological Features of MSI Tumors. A summary of the main clinicopathological features of MSI cancers compared to MSS cancers is shown in Table 2. In the majority of cases, tumors with MSI were located in the right colon compared to
TABLE 1: Clinicopathological features of 330 patients.

\begin{tabular}{|c|c|}
\hline Variables & $N(\%)$ \\
\hline Age (mean) & 54.62 years \pm 14.7 \\
\hline$<50$ & $123(37.3 \%)$ \\
\hline$>50$ & $207(62.7 \%)$ \\
\hline \multicolumn{2}{|l|}{ Sex } \\
\hline Female & $156(47.3 \%)$ \\
\hline Male & $174(52.7 \%)$ \\
\hline \multicolumn{2}{|l|}{ Tumor site } \\
\hline Right colon & $76(23.0 \%)$ \\
\hline Left colon & $254(77.0 \%)$ \\
\hline \multicolumn{2}{|l|}{ Histologic subtype } \\
\hline Adenocarcinoma & $278(84.2 \%)$ \\
\hline Mucinous adenocarcinoma & $29(8.8 \%)$ \\
\hline Others & $23(7 \%)$ \\
\hline \multicolumn{2}{|l|}{ Histologic grade } \\
\hline Well & $173(52.4 \%)$ \\
\hline Moderate & $128(38.8 \%)$ \\
\hline Poor & $28(8.5 \%)$ \\
\hline Unknown & $1(0.3 \%)$ \\
\hline \multicolumn{2}{|l|}{ Perineural invasion } \\
\hline Yes & $27(8.2 \%)$ \\
\hline No & $272(82.4 \%)$ \\
\hline Unknown & $31(9.4 \%)$ \\
\hline \multicolumn{2}{|l|}{ Vascular invasion } \\
\hline Yes & $39(11.8 \%)$ \\
\hline No & $291(88.2 \%)$ \\
\hline \multicolumn{2}{|l|}{ Disease stage } \\
\hline I & $28(8.5 \%)$ \\
\hline II & $161(48.4 \%)$ \\
\hline III & $73(22.1 \%)$ \\
\hline IV & $68(20.6 \%)$ \\
\hline \multicolumn{2}{|l|}{ MSI status } \\
\hline MSS & $293(88.8 \%)$ \\
\hline MSI & $37(11.2 \%)$ \\
\hline
\end{tabular}

$N$ : number of cases; SD: standard deviation; MSS: microsatellite stable; MSI: microsatellite instability.

MSS tumors $(P \leq 0.001)$. Male gender was significantly associated with MSI tumors ( $72.0 \%$ versus $28.0 \%, P=0.04)$. Moreover, MSI tumors were more commonly found in patients under 57 years $(P=0.05)$. As for distant metastases, no patient with MSI status had distant metastases at the time of diagnostic, and the difference was statistically significant $(P=0.03)$. Regarding pathologic disease stage, stage I-II were more common in MSI tumors $(P=0.02)$. Histologically, the degree of differentiation was associated with MSI tumors and the difference was statistically significant $(P=0.04)$. In contrast, no significant correlation was found between MSI tumors and histologic subtype, T stage, vascular invasion, perineural invasion, family history of colon cancer, and RAS mutation. 


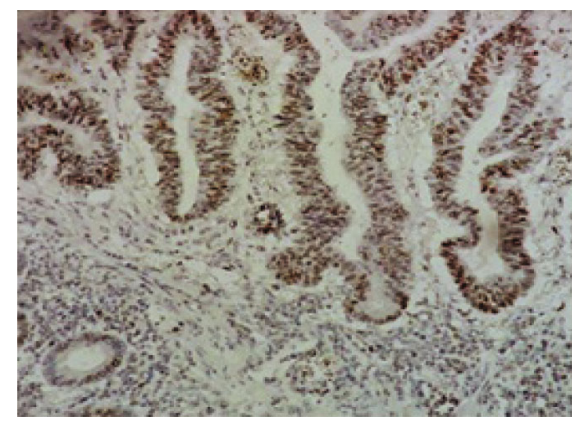

(a)

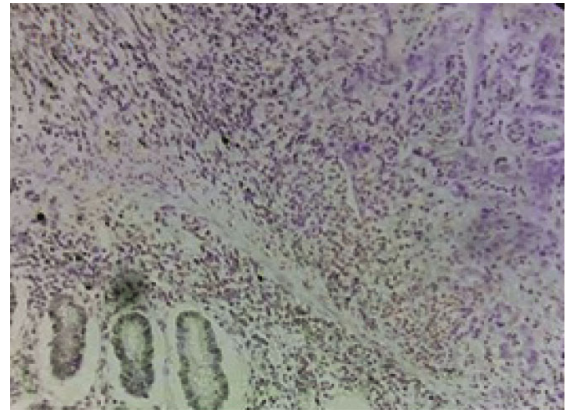

(b)

FIGURE 2: Immunohistochemical staining for MMR proteins. (a) Overexpression of MLH1 protein in colon adenocarcinoma (magnification 200x). (b) MLH1 loss in colon adenocarcinoma (magnification 200x).
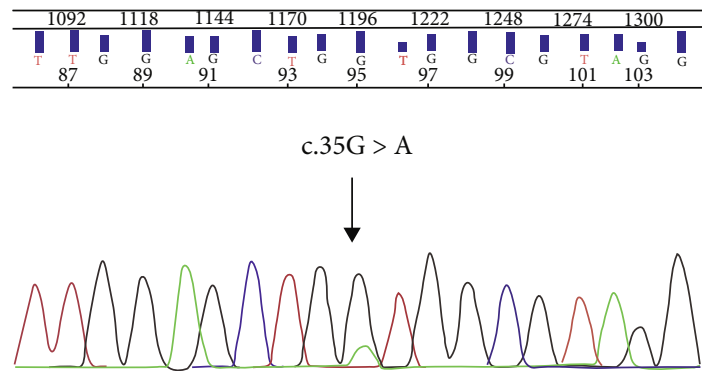

(a)
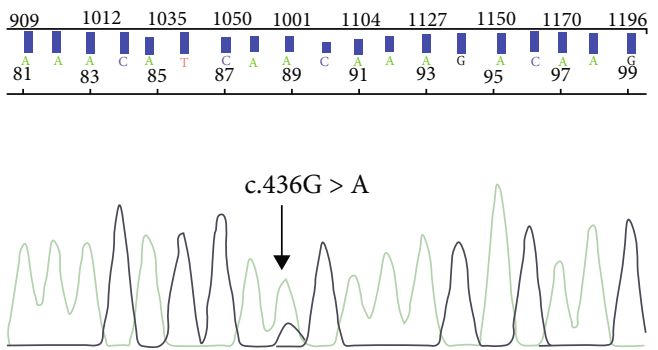

(b)

Figure 3: Direct sequencing chromatograms. (a) KRAS exon 2 mutation (c.35G>A, p.G12D change), (b) KRAS exon 4 mutation (c.436 G>A, p.A146T change).

3.4. Nodal Counts in Stage I-IV Colon Cancer: Comparison of the Total Lymph Nodes (LNs), Metastatic LNs, and LNR according to MSI Status. When analyzing our cohort, MSI tumors were associated with a significantly higher total LN count (mean: 23.440 vs. 19.075, $P=0.04$ ). However, the number of positive LNs was significantly lower in MSI patients (mean: 0.28 vs. $1.34, P=0.007$ ). The LNR was also lower in MSI tumors, and the difference was statistically significant ( 0.01 vs. $0.08, P=0.01)$. The lymph node characteristics of MSI tumors are compiled in Table 3.

When analyzing our cohort, MSI tumors were associated with a significantly higher total LN count (mean: 21.08 vs. $17.68, P=0.04$ ). However, the number of positive LNs was significantly lower in MSI patients (mean: 0.62 vs. 1.33 , $P=0.03)$. The LNR was also lower in MSI tumors, and the difference was statistically significant (0.03 vs. 0.08 , $P=0.02$ ). The lymph node characteristics of MSI tumors are compiled in Table 3.

3.5. Clinicopathological Features of Patients with Right Colon Cancer and Left Colon Cancer. Table 4 shows the basic characteristics between the right colon cancer (RCC) and the left colon cancer (LCC). The mean age of right-sided tumors was significantly younger than that of left-sided tumors (52.5 and 58.5 years old, respectively, $P=0.01$ ). Patients with RCC were significantly more likely to be male in our study $(P=0.03)$. Regarding pathologic grade, moderate differentiated tumors were less common in RCC than in LCC patients
$(P=0.05)$. In addition, a mucinous adenocarcinoma subtype was significantly associated with RCC tumors than with LCC tumors $(P=0.01)$. At all stages, the mean of harvested lymph nodes was significantly higher in RC tumors than in LCC tumors (22.2 vs. 18.17, $P=0.01)$. More LCC had a higher incidence of metastatic disease at diagnosis ( $15.8 \%$ vs. $6.3 \%$, $P=0.05)$. In this study, $30.2 \%$ of RCC patients were microsatellite unstable compared to $5.9 \%$ of LCC patients $(P \leq 0.001)$. The frequency of RAS mutation did not differ between RCC tumors and LCC tumors in our study.

3.6. Comparison of Clinicopathological Features between Patients Less than 58 Years Old and Older. As shown in Table 5, we divided the patients into two groups: patients who were 57 years old and less and patients more than 57 years old. The proportion of right colon cancers was higher in patients 57 years old and less than in older patients $(P=0.02)$. The proportion of poorly differentiated tumors was higher in the youngest age subgroup than in the oldest (11.8\% vs. 5.0\%, $P=0.05)$. Interestingly, the presence of a family history of colon cancer was significantly associated with the youngest age subgroup $(P=0.02)$. The youngest age subgroup had more nodes examined than the oldest age subgroup $(P<0.001)$. Moreover, $90.7 \%$ of the youngest patients have more than 12 lymph nodes collected, compared with $80.6 \%$ in the oldest patients $(P=0.04)$. Generally, the MSI status was also significantly correlated with the youngest age subgroup in our series $(P=0.01)$. 
TABLE 2: Clinicopathological features of MSI cancers.

\begin{tabular}{|c|c|c|c|}
\hline Characteristics & Defect MMR & Intact MMR & $P$ value \\
\hline \multicolumn{4}{|l|}{ Age } \\
\hline Mean & $51.5( \pm 14.18)$ & $55.0( \pm 15.45)$ & 0.1 \\
\hline$\leq 57$ & $26(70.3 \%)$ & $144(49.1 \%)$ & \multirow{2}{*}{0.01} \\
\hline$\geq 57$ & $11(29.7 \%)$ & $149(50.9 \%)$ & \\
\hline \multicolumn{4}{|l|}{ Gender } \\
\hline Female & $10(27.0 \%)$ & $146(49.8 \%)$ & \multirow{2}{*}{0.007} \\
\hline Male & $27(73.0 \%)$ & $147(50.2 \%)$ & \\
\hline \multicolumn{4}{|l|}{ Tumor site } \\
\hline Right colon & $23(62.2 \%)$ & $53(18.1 \%)$ & \multirow{2}{*}{0.000} \\
\hline Left colon & $14(37.8 \%)$ & $240(81.9 \%)$ & \\
\hline \multicolumn{4}{|l|}{ Histologic subtype } \\
\hline Adenocarcinoma & $30(81.8 \%)$ & $248(84.6 \%)$ & \multirow{3}{*}{0.1} \\
\hline $\begin{array}{l}\text { Mucinous } \\
\text { adenocarcinoma }\end{array}$ & $6(16.2 \%)$ & $23(7.8 \%)$ & \\
\hline Others & $1(2.7 \%)$ & $22(7.5 \%)$ & \\
\hline \multicolumn{4}{|l|}{ Histologic grade } \\
\hline Well & $15(40.5 \%)$ & $208(71.5 \%)$ & \multirow{2}{*}{0.000} \\
\hline Poor & $22(59.5 \%)$ & $83(28.5 \%)$ & \\
\hline \multicolumn{4}{|l|}{ Vascular invasion } \\
\hline Yes & $7(18.9 \%)$ & $32(10.9 \%)$ & \multirow{2}{*}{0.1} \\
\hline No & $30(81.1 \%)$ & $261(89.1 \%)$ & \\
\hline \multicolumn{4}{|l|}{ Perineural invasion } \\
\hline Yes & $2(5.7 \%)$ & $25(9.5 \%)$ & \multirow{2}{*}{0.3} \\
\hline No & $33(94.3 \%)$ & $239(90.5 \%)$ & \\
\hline \multicolumn{4}{|c|}{ Family history of cancer } \\
\hline Yes & $3(8.1 \%)$ & $9(3.1 \%)$ & \multirow{2}{*}{0.1} \\
\hline No & $34(91.9 \%)$ & $284(96.9 \%)$ & \\
\hline \multicolumn{4}{|l|}{ Tumor stage (T) } \\
\hline $\mathrm{T} 1$ & $0(0.0 \%)$ & $4(1.3 \%)$ & \multirow{4}{*}{0.5} \\
\hline $\mathrm{T} 2$ & $5(13.5 \%)$ & $47(16.2 \%)$ & \\
\hline $\mathrm{T} 3$ & $25(67.6 \%)$ & $173(59.5 \%)$ & \\
\hline $\mathrm{T} 4$ & $7(18.9 \%)$ & $67(23.0 \%)$ & \\
\hline \multicolumn{4}{|c|}{ Distant metastases (M) } \\
\hline M0 & $36(97.3 \%)$ & $222(76.8 \%)$ & \multirow{2}{*}{0.001} \\
\hline M1 & $1(2.7 \%)$ & $67(23.2 \%)$ & \\
\hline \multicolumn{4}{|l|}{ Disease stages } \\
\hline I-II & $28(75.7 \%)$ & $160(54.6 \%)$ & \multirow{2}{*}{0.01} \\
\hline III-IV & $9(24.3 \%)$ & $133(45.4 \%)$ & \\
\hline \multicolumn{4}{|l|}{ RAS mutation } \\
\hline Presence & $6(15.8 \%)$ & $32(84.2 \%)$ & \multirow{2}{*}{0.09} \\
\hline Absence & $12(15.4 \%)$ & $66(84.6 \%)$ & \\
\hline
\end{tabular}

MMR: mismatch repair; SD: standard deviation.

\section{Survival Analysis}

The median length of the follow-up period was 30.00 months (range 0-117 months). From 330 patients' colon cancer, 33 patients (10\%) developed distant metastasis during the follow-up period. The time to metastasis ranged from 2 to 46 months (median $=11.00$ months). The most common
TABLE 3: Comparison of lymph node features according to MSI status.

\begin{tabular}{|c|c|c|c|}
\hline & $\begin{array}{c}\text { MSI } \\
(n=37)\end{array}$ & $\begin{array}{c}\text { MSS } \\
(n=293)\end{array}$ & $P$ value $(95 \% \mathrm{CI})$ \\
\hline \multicolumn{4}{|l|}{ Total node } \\
\hline Mean (SD) & $21.08( \pm 9.94)$ & $17.68( \pm 9.54)$ & $\begin{array}{c}P=0.04 \\
(0.053 \text { to } 6.74)\end{array}$ \\
\hline Positive node & $0.62( \pm 1.49)$ & $1.33( \pm 3.10)$ & $\begin{array}{c}P=0.03 \\
(-1.35 \text { to }-0.005)\end{array}$ \\
\hline $\begin{array}{l}\text { Lymph node } \\
\text { ratio }\end{array}$ & $0.03( \pm 0.08)$ & $0.08( \pm 0.27)$ & $\begin{array}{c}P=0.02 \\
(-0.097 \text { to }-0.005)\end{array}$ \\
\hline
\end{tabular}

MSI: microsatellite instability; MSS: microsatellite stable; SD: standard deviation.

metastatic site was peritoneum $(n=13,43.3 \%)$ followed by the lung $(n=11,36.6 \%)$, liver $(n=10,33.3 \%)$, Os $(n=1$, $3.3 \%)$, ovary $(n=1,3.3 \%)$, distant lymph nodes $(n=1$, $3.3 \%)$, and head $(n=1,3.3 \%)$. Regarding recidivism, 9 of 330 patients developed a disease relapse. The median length of recidivism was 11.0 months (range 0-29 months).

According to our findings in our cohort, we observed a better prognosis of MSI patients compared with that of MSS patients and the difference was statistically significant ( $109.71 \%$ vs. $74.08 \%, P=0.001$ ) (Figure $4(\mathrm{a})$ ). The variables associated with the OS are shown in Table 6. On univariate analysis, the male gender was significantly associated with OS $(P=0.003)$ (Figure 4(b)). The OS of patients with stage III-IV disease was significantly lower than that of patients with stage I-II disease $(63.85 \%$ vs. $89.48 \%, P=0.000)$ (Figure 4(d)). Moreover, right localization and male gender were found to be statistically significant predictors of poor outcomes in our population (Figure 4(c)). Interestingly, we stratified the LNR into 2 subgroups based on the mean value of 0.07 , and we documented that the higher value of LNR was a better predictor of prognosis $(P=0.03)$ (Figure $4(\mathrm{e})$ ).

Cox proportional hazard regression was performed for factors that were significant in univariate analysis $(P<0.05)$ (MSI status, gender, tumor site, disease stage, and LNR). Multivariate analysis revealed that female gender, left localization, and III-IV were the independent poor prognostic factors for OS in colon cancer Table 6.

\section{Discussion}

In the present study, we aimed to evaluate the impact of MSI status and clinicopathological features on overall survival in the Moroccan population. Our findings suggest that MSI tumors occur in $11.2 \%$ in the FES population which is similar to previous studies which confirmed that the rate of dMMR tumors in colon cancer is between $10 \%$ and $15 \%[12,13]$. In addition, we observed that MSI tumors were commonly found in younger patients $(\leq 57)$ and in the right colon as reported in the literature $[13,14]$. In line with other studies $[10,14,15]$, we found that MSI tumors were mostly diagnosed in stage II (75.7\%). This finding could explain the more favorable prognosis of MSI subtype, described in several studies $[14,16]$. 
TABLE 4: Comparison of clinicopathological characteristics between the right colon and left colon.

\begin{tabular}{|c|c|c|c|}
\hline Characteristics & Right colon & Left colon & $P$ value \\
\hline \multicolumn{4}{|l|}{ Age } \\
\hline Mean (SD) & $51.4( \pm 15.7)$ & $55.5( \pm 15.0)$ & 0.03 \\
\hline$\leq 57$ & $47(61.8 \%)$ & $123(48.4 \%)$ & \multirow{2}{*}{0.002} \\
\hline$\geq 57$ & $29(38.2 \%)$ & $131(51.6 \%)$ & \\
\hline \multicolumn{4}{|l|}{ Gender } \\
\hline Female & $28(36.8 \%)$ & $128(50.4 \%)$ & \multirow{2}{*}{0.02} \\
\hline Male & $48(63.2 \%)$ & $126(49.6 \%)$ & \\
\hline \multicolumn{4}{|l|}{ Histologic subtype } \\
\hline Adenocarcinoma & $59(77.6 \%)$ & $219(86.2 \%)$ & \multirow{3}{*}{0.04} \\
\hline $\begin{array}{l}\text { Mucinous } \\
\text { adenocarcinoma }\end{array}$ & $12(15.8 \%)$ & $17(6.7 \%)$ & \\
\hline Others & $5(6.6 \%)$ & $18(7.1 \%)$ & \\
\hline \multicolumn{4}{|l|}{ Histologic grade } \\
\hline Well & $42(55.3 \%)$ & $131(51.8 \%)$ & \multirow{3}{*}{0.05} \\
\hline Moderate & $26(34.2 \%)$ & $102(40.3 \%)$ & \\
\hline Poor & $8(10.5 \%)$ & $20(7.9 \%)$ & \\
\hline \multicolumn{4}{|l|}{ Vascular invasion } \\
\hline Yes & $14(18.4 \%)$ & $25(9.8 \%)$ & \multirow{2}{*}{0.03} \\
\hline No & $62(81.6 \%)$ & $229(90.2 \%)$ & \\
\hline \multicolumn{4}{|l|}{ Perineural invasion } \\
\hline Yes & $7(9.2 \%)$ & $20(9.0 \%)$ & \multirow{2}{*}{0.5} \\
\hline No & $69(90.8 \%)$ & $203(91.0 \%)$ & \\
\hline \multicolumn{4}{|l|}{ Total lymph node } \\
\hline Mean (SD) & $20.67( \pm 11.28)$ & $17.28( \pm 8.85)$ & 0.01 \\
\hline $\mathrm{LN}>12$ & $53(74.7 \%)$ & $82(46.7 \%)$ & \multirow{2}{*}{0.000} \\
\hline $\mathrm{LN}<12$ & $18(25.3 \%)$ & $105(53.3 \%)$ & \\
\hline \multicolumn{4}{|c|}{ Family history of cancer } \\
\hline Yes & $5(6.6 \%)$ & $7(2.8 \%)$ & \multirow{2}{*}{0.1} \\
\hline No & $71(93.4 \%)$ & $247(97.2 \%)$ & \\
\hline \multicolumn{4}{|l|}{ Tumor stage $(\mathrm{T})$} \\
\hline $\mathrm{T} 1$ & $1(1.3 \%)$ & $3(1.2 \%)$ & \multirow{4}{*}{0.8} \\
\hline $\mathrm{T} 2$ & $10(13.2 \%)$ & $42(16.7 \%)$ & \\
\hline T3 & $48(63.2 \%)$ & $150(59.5 \%)$ & \\
\hline $\mathrm{T} 4$ & $17(22.4 \%)$ & $57(22.6 \%)$ & \\
\hline \multicolumn{4}{|c|}{ Distant metastases (M) } \\
\hline M0 & $68(91.9 \%)$ & $190(75.4 \%)$ & \multirow{2}{*}{0.001} \\
\hline M1 & $6(8.1 \%)$ & $68(24.6 \%)$ & \\
\hline \multicolumn{4}{|l|}{ Disease stages } \\
\hline I & $9(11.8 \%)$ & $19(7.5 \%)$ & \multirow{4}{*}{0.002} \\
\hline II & $45(59.2 \%)$ & $116(45.7 \%)$ & \\
\hline III & $17(22.4 \%)$ & $56(22.0 \%)$ & \\
\hline IV & $5(6.6 \%)$ & $63(24.8 \%)$ & \\
\hline \multicolumn{4}{|l|}{ MSI status } \\
\hline MSI & $23(30.3 \%)$ & $14(5.5 \%)$ & 00000 \\
\hline MSS & $53(69.7 \%)$ & $240(94.5 \%)$ & 0.0000 \\
\hline RAS mutation & & & \\
\hline Presence & $13(34.2 \%)$ & $25(65.8 \%)$ & 04 \\
\hline Absence & $22(28.2 \%)$ & $56(71.8 \%)$ & 0.4 \\
\hline
\end{tabular}

SD: standard deviation; LN: lymph node; MSI: microsatellite instability; MSS: microsatellite stable.
TABLE 5: Comparison of clinicopathological parameters between patients less than 57 years old and older patients.

\begin{tabular}{|c|c|c|c|}
\hline Characteristics & $\leq 57$ & $\geq 57$ & $P$ value \\
\hline \multicolumn{4}{|l|}{ Gender } \\
\hline Female & $88(51.8 \%)$ & $68(42.5 \%)$ & \multirow{2}{*}{0.05} \\
\hline Male & $82(48.2 \%)$ & $92(57.5 \%)$ & \\
\hline \multicolumn{4}{|l|}{ Tumor site } \\
\hline Right colon & $47(27.6 \%)$ & $29(18.1 \%)$ & \multirow{2}{*}{0.02} \\
\hline Left colon & $123(72.4 \%)$ & $131(81.9 \%)$ & \\
\hline \multicolumn{4}{|l|}{ Histologic subtype } \\
\hline Adenocarcinoma & $138(81.2 \%)$ & $140(87.5 \%)$ & \multirow{3}{*}{0.07} \\
\hline $\begin{array}{l}\text { Mucinous } \\
\text { adenocarcinoma }\end{array}$ & $15(8.8 \%)$ & $14(8.8 \%)$ & \\
\hline Others & $17(10.0 \%)$ & $6(3.8 \%)$ & \\
\hline \multicolumn{4}{|l|}{ Histologic grade } \\
\hline Well & $88(52.1 \%)$ & $85(53.1 \%)$ & \multirow{3}{*}{0.05} \\
\hline Moderate & $61(36.1 \%)$ & $67(41.9 \%)$ & \\
\hline Poor & $20(11.8 \%)$ & $8(5.0 \%)$ & \\
\hline \multicolumn{4}{|l|}{ Vascular invasion } \\
\hline Yes & $16(9.4 \%)$ & $23(14.4 \%)$ & \multirow{2}{*}{0.1} \\
\hline No & $154(90.6 \%)$ & $137(85.6 \%)$ & \\
\hline \multicolumn{4}{|l|}{ Perineural invasion } \\
\hline Yes & $14(9.1 \%)$ & $13(9.0 \%)$ & \multirow{2}{*}{0.5} \\
\hline No & $140(90.9 \%)$ & $132(91.0 \%)$ & \\
\hline \multicolumn{4}{|l|}{ Total lymph node } \\
\hline Mean (SD) & $\begin{array}{c}24.5 \\
( \pm 10.30)\end{array}$ & $\begin{array}{c}17.9 \\
( \pm 9.06)\end{array}$ & 0.000 \\
\hline $\mathrm{LN}>12$ & $68(90.7 \%)$ & $58(80.6 \%)$ & \multirow{2}{*}{0.04} \\
\hline $\mathrm{LN}<12$ & $7(9.3 \%)$ & $14(19.4 \%)$ & \\
\hline \multicolumn{4}{|c|}{ Family history of cancer } \\
\hline Yes & $9(5.3 \%)$ & $3(1.9 \%)$ & \multirow{2}{*}{0.02} \\
\hline No & $161(94.7 \%)$ & $157(98.1 \%)$ & \\
\hline \multicolumn{4}{|l|}{ Tumor stage $(\mathrm{T})$} \\
\hline $\mathrm{T} 1$ & $3(0.0 \%)$ & $1(0.6 \%)$ & \multirow{4}{*}{0.4} \\
\hline $\mathrm{T} 2$ & $22(13.1 \%)$ & $30(18.8 \%)$ & \\
\hline $\mathrm{T} 3$ & $101(60.1 \%)$ & $97(60.6 \%)$ & \\
\hline $\mathrm{T} 4$ & $42(25.0 \%)$ & $32(20.0 \%)$ & \\
\hline \multicolumn{4}{|c|}{ Distant metastases (M) } \\
\hline M0 & $134(79.8 \%)$ & $124(78.5 \%)$ & \multirow{2}{*}{0.4} \\
\hline M1 & $34(20.2 \%)$ & $34(21.5 \%)$ & \\
\hline \multicolumn{4}{|l|}{ Disease stages } \\
\hline I & $13(7.6 \%)$ & $15(9.4 \%)$ & \multirow{4}{*}{0.6} \\
\hline II & $80(47.1 \%)$ & $81(50.6 \%)$ & \\
\hline III & $42(24.7 \%)$ & $31(19.4 \%)$ & \\
\hline IV & $35(20.6 \%)$ & $33(20.6 \%)$ & \\
\hline \multicolumn{4}{|l|}{ MSI status } \\
\hline MSI & $26(15.3 \%)$ & $11(6.9 \%)$ & \multirow{2}{*}{0.01} \\
\hline MSS & $144(84.7 \%)$ & $149(93.1 \%)$ & \\
\hline RAS mutation & & & \\
\hline Presence & $16(46.8 \%)$ & $22(57.9 \%)$ & 03 \\
\hline Absence & $36(42.1 \%)$ & $41(53.2 \%)$ & 0.0 \\
\hline
\end{tabular}

SD: standard deviation; LN: lymph node; MSI: microsatellite instability; MSS: microsatellite stable. 


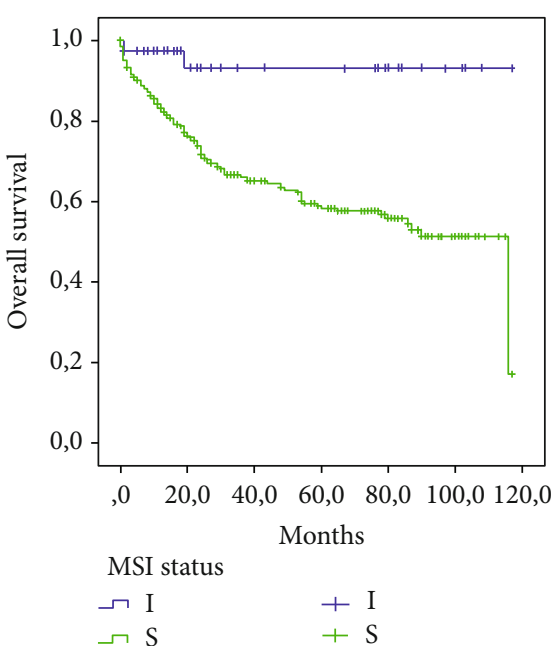

(a)

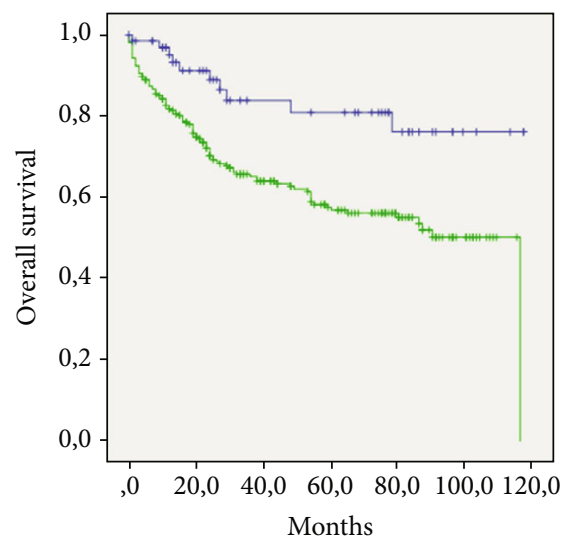

Tumor location

$\neg$ Right $+1,0$

(c)

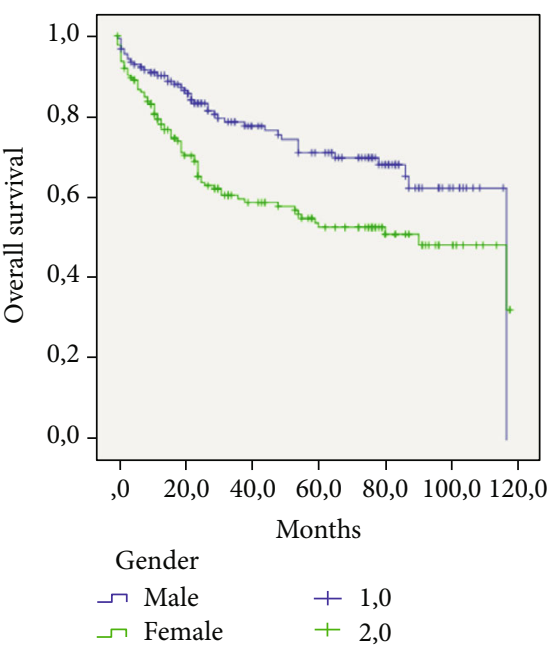

(b)

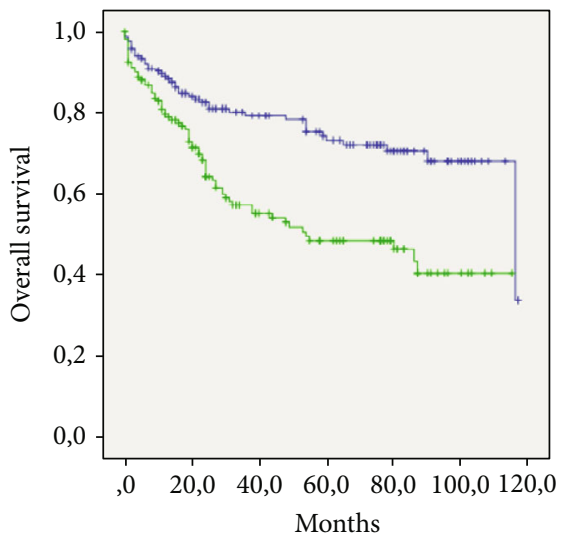

Disease stage

$\begin{array}{ll}\neg \text { I-II } & +1,0 \\ \neg \text { III-IV } & +2,0\end{array}$

(d)

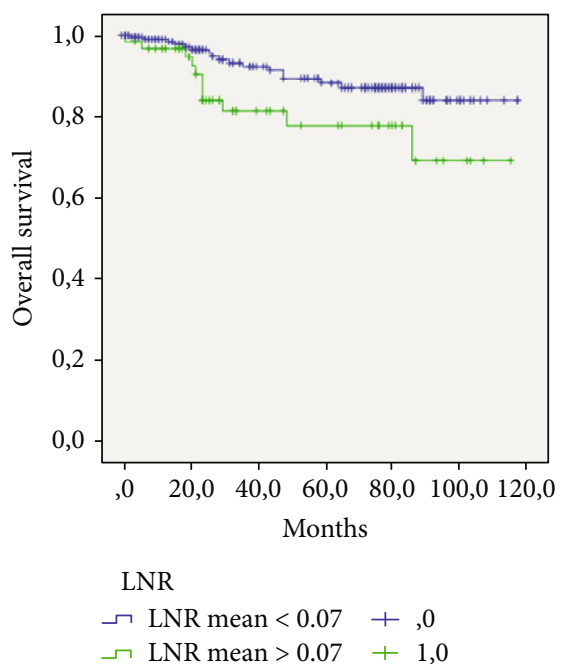

(e)

Figure 4: The Kaplan-Meier overall survival curve of the 330 colon cancer patients according to different variables: (a) MSI status, (b) Gender, (c) tumor localization, (d) disease stage, and (e) LNR.

Our study has been able to demonstrate a positive relationship between MSI tumors and total lymph node count. Recently, Tian et al. [13] found the same observation in a cohort of 1250 stage II-IV CRC cases. These findings are a possible explanation for the high immune response observed in MSI tumors [17, 18]. In addition, the patients enrolled in our study showed a significantly lower rate of positive lymph node. The low lymph node ratio (LNR) was also associated with MSI tumors in all stages of CC. This finding is in line with the results reported by Ghanipour et al. [15] in a recent publication which indicated a relevant association between MSI high and low rate of LNR. A previous study documented that low LNR is a predictor of good outcome $[19,20]$. These results explain the better survival of patients with MSI status. Despite better outcomes, our study showed a positive correlation between MSI status and poor differentiation, which is reported as a factor of poor prognosis. This result is in agreement with several reports [21-23].
In our study, from 200 patients, we did not find any $B R A F$ mutation in both MSI and MSS tumors. This result confirms the rarity of the V600E mutation in colon cancer, epically in the Moroccan population [17].

On the other hand, many investigators demonstrate that RCC and LCC show clinicopathological and molecular differences. In the present study, RCC tumors are more likely mucinous, commonly observed in younger patients, had less metastasis disease, and showed frequently MSI status. In univariate and multivariate analyses, we found that RCC had better OS than LCC and the significant difference of the Cox hazard ratio between the two subgroups was reported in our series $(P=0.05)$. Some studies have documented that overall survival is generally worse in the right colon compared to the left colon [24, 25], while others revealed no difference [18]. The molecular profile of right colon cancer characterized by the presence of RAS/BRAF mutation which correlated with worse outcome [26]. In the present study, there was no association between RC location and RAS mutation. 
TABLE 6: The clinical variables associated with overall survivals in the 330 colon cancer patients.

\begin{tabular}{|c|c|c|c|c|}
\hline \multirow{2}{*}{ Variables } & \multicolumn{2}{|c|}{ Univariate analysis } & \multicolumn{2}{|c|}{ Multivariate analysis } \\
\hline & Mean OS months (95\% CI) & $P$ value & Hazard ratio (95\% CI) & $P$ value \\
\hline \multicolumn{5}{|l|}{ Age (yr) } \\
\hline$\geq 57$ & $79.77(71.86-87.68)$ & \multirow{2}{*}{0.4} & & \\
\hline$<57$ & $75.08(66.59-83.56)$ & & & \\
\hline \multicolumn{5}{|l|}{ Gender } \\
\hline Female & $69.09(60.91-77.28)$ & \multirow{2}{*}{0.003} & \multirow{2}{*}{$1.52(1.03-3.08)$} & \multirow{2}{*}{0.03} \\
\hline Male & $86.19(78.37-94.01)$ & & & \\
\hline \multicolumn{5}{|l|}{ Tumor site } \\
\hline Right side & 97.07 (85.97-108.17) & \multirow{2}{*}{0.001} & \multirow{2}{*}{$1.79(0.98-3.71)$} & \multirow{2}{*}{0.05} \\
\hline Left side & $72.72(66.27-79.16)$ & & & \\
\hline \multicolumn{5}{|l|}{ Tumor stage } \\
\hline I-II & 89.48 (82.28-96.68) & \multirow{2}{*}{0.000} & \multirow{2}{*}{$1.49(1.29-2.82)$} & \multirow{2}{*}{0.001} \\
\hline III-IV & $63.85(55.48-72.22)$ & & & \\
\hline \multicolumn{5}{|l|}{ LNR (mean) } \\
\hline 1 & $105.50(100.36-110.64)$ & \multirow{2}{*}{0.03} & \multirow{2}{*}{$2.04(0.80-5.18)$} & \multirow{2}{*}{0.1} \\
\hline 2 & $92.38(80.66-104.10)$ & & & \\
\hline \multicolumn{5}{|l|}{ TLN } \\
\hline$\geq 12$ & $104.77(96.26-113.27)$ & \multirow{2}{*}{0.6} & & \\
\hline$<12$ & $101.72(95.73-107.71)$ & & & \\
\hline \multicolumn{5}{|c|}{ Perineural invasion } \\
\hline Yes & $74.80(58.63-90.96)$ & \multirow{2}{*}{0.6} & & \\
\hline No & $82.64(76.56-88.72)$ & & & \\
\hline \multicolumn{5}{|c|}{ Vascular invasion } \\
\hline Yes & $88.47(73.13-103.81)$ & \multirow{2}{*}{0.1} & & \\
\hline No & $75.87(69.73-82.02)$ & & & \\
\hline \multicolumn{5}{|l|}{ MSI status } \\
\hline MSI & $109.71(99.85-119.58)$ & \multirow{2}{*}{0.001} & \multirow{2}{*}{$0.11(0.06-1.04)$} & \multirow{2}{*}{0.05} \\
\hline MSS & $74.08(68.00-80.16)$ & & & \\
\hline RAS mutatio & & & & \\
\hline Presence & $52.52(40.918-64.133)$ & 05 & & \\
\hline Absence & $56.75(42.057-71.452)$ & 0.3 & & \\
\hline
\end{tabular}

CI: confidence interval; OS: overall survival; LNR: lymph node ratio; TLN: total lymph node; MSI: microsatellite instability; MSS: microsatellite stable.

The relationship between MSI tumors and OS is unclear according to Elias et al. [19]. In our study, there was an improved OS in patients with stage I-IV and MSI status. In line with our findings, previous studies have demonstrated that MSI tumors have a better clinical outcome [27]. The prognosis value of MSI tumors could be explained by the low rate of metastasis at diagnosis observed in these tumors, which is confirmed in our study. Indeed, Ferri et al. [21] have reported that the MSI patients with locally advanced colorectal cancers had a better prognosis.

According to many studies, low LNR was proven to be a strong prognostic factor of survival $[20,26]$. In addition, Emterling et al. [22] documented a significant association between higher LNR and reduced OS and time to recurrence in patients with stage III of CRC. In our study, we found an improved OS in patients with low LNR, with a significant statistical difference on univariate analysis
$(P=0.03)$, although TLN was not found to be an independent prognostic factor in our series on both univariate and multivariate analyses. In line with our results, Jass et al. [23] reported no significant difference of the Cox hazard ratio between OS and TLN.

Reviewing our data, we found that patients who were diagnosed with stage III-IV disease had the highest risk of death compared to those with stage I-II disease. Our results are in agreement with the finding recently reported by Weiss et al. [24]. Interestingly and contrary to previous studies $[28,29]$, we documented a strong significant correlation between male gender and OS in all stages, while other reports did not find any difference in survival between genders [30, 31]. Our result may be related to the proportions of colon cancer patients included in our study and the stronger correlation observed between MSI status and male gender. Moreover, the multivariate 
analysis revealed that MSI status, right localization, stage I-II, and male gender were the most significant prognostic factors for overall survival in Moroccan patients.

\section{Conclusion}

In summary, our study demonstrates that patients with MSI status have particular clinicopathological features like TLN, LNR, poor differentiation, right colon, locally advanced tumors, male gender, and younger patients. The results of survival analysis indicate that MSI status was not predictive of improved overall survival in our context with a lower statistical significance $(P=0.05)$. After cox regression analysis, the right localization of the tumor, I-II stage disease, and male gender showed a trend toward a better prognosis in our population.

\section{Data Availability}

The datasets used/or analyzed during the current study are available from the corresponding author on reasonable request.

\section{Conflicts of Interest}

The authors report no conflicts of interest.

\section{Authors' Contributions}

The authors alone are responsible for the content and writing of the paper.

\section{References}

[1] E. Ryan, K. Sheahan, B. Creavin, H. M. Mohan, and D. C. Winter, "The current value of determining the mismatch repair status of colorectal cancer: a rationale for routine testing," Critical Reviews in Oncology/Hematology, vol. 116, pp. 38-57, 2017.

[2] W. Chen, B. J. Swanson, and W. L. Frankel, "Molecular genetics of microsatellite-unstable colorectal cancer for pathologists," Diagnostic Pathology, vol. 12, no. 1, 2017.

[3] H. Kawakami, A. Zaanan, and F. A. Sinicrope, "Microsatellite instability testing and its role in the management of colorectal cancer," Current Treatment Options in Oncology, vol. 16, 2015.

[4] M. Svrcek, P. Cervera, R. Hamelin, O. Lascols, A. Duval, and J. F. Fléjou, "Colorectal cancer : news roles for the pathologists in the era of molecular biology and "targeted" therapies," Revue Francophone des Laboratoires, vol. 2011, no. 428, pp. 29-41, 2011.

[5] G. Thodi, F. Fostira, R. Sandaltzopoulos et al., "Screening of the DNA mismatch repair genes $M L H 1, M S H 2$ and MSH6 in a Greek cohort of Lynch syndrome suspected families," BMC Cancer, vol. 10, 2010.

[6] P. Peltomäki, "Lynch syndrome genes," Familial Cancer, vol. 4, pp. 227-232, 2005.

[7] K. Söreide, E. A. Janssen, H. Söiland, H. Körner, and J. P. Baak, "Microsatellite instability in colorectal cancer," British Journal of Surgery, vol. 93, pp. 395-406, 2006.
[8] F. Pagès, A. Berger, M. Camus et al., "Effector memory T cells, early metastasis, and survival in colorectal cancer," New England Journal of Medicine, vol. 353, pp. 2654-2666, 2005.

[9] J. Guinney, R. Dienstmann, X. Wang et al., "The consensus molecular subtypes of colorectal cancer," Nature Medicine, vol. 21, no. 11, pp. 1350-1356, 2015.

[10] A. Malesci, L. Laghi, P. Bianchi et al., "Reduced likelihood of metastases in patients with microsatellite-unstable colorectal cancer," Clinical Cancer Research, vol. 13, no. 22, pp. 38313840, 2007.

[11] C. C. J. M. Simons, L. A. E. Hughes, K. M. Smits et al., “A novel classification of colorectal tumors based on microsatellite instability, the CpG island methylator phenotype and chromosomal instability: implications for prognosis," Annals of Oncology, vol. 24, no. 8, pp. 2048-2056, 2013.

[12] J. Taieb, K. Le Malicot, Q. Shi et al., "Prognostic value of BRAF and KRAS mutations in MSI and MSS stage III colon cancer," vol. 109, Article ID djw272, 2017.

[13] S. Tian, P. Roepman, V. Popovici et al., "A robust genomic signature for the detection of colorectal cancer patients with microsatellite instability phenotype and high mutation frequency," The Journal of Pathology, vol. 228, pp. 586-595, 2012.

[14] J. M. Bae, J. H. Kim, and G. H. Kang, "Molecular subtypes of colorectal cancer and their clinicopathologic features, with an emphasis on the serrated neoplasia pathway," Archives of Pathology \& Laboratory Medicine, vol. 140, 2016.

[15] L. Ghanipour, K. Jirström, M. Sundström, B. Glimelius, and H. Birgisson, "Associations of defect mismatch repair genes with prognosis and heredity in sporadic colorectal cancer," European Journal of Surgical Oncology, vol. 43, no. 2, pp. 311-321, 2017.

[16] G. M. Nash, M. Gimbel, A. M. Cohen et al., "KRAS mutation and microsatellite instability: two genetic markers of early tumor development that influence the prognosis of colorectal cancer," Annals of Surgical Oncology, vol. 17, no. 2, pp. 416424, 2010.

[17] J. M. Michael-Robinson, G. L. Radford-Smith, A. E. BiemerHüttmann et al., "Tumour infiltrating lymphocytes and apoptosis are independent features in colorectal cancer stratified according to microsatellite instability status," Gut, vol. 48, no. 3, pp. 360-366, 2001.

[18] S. M. Phillips, A. Banerjea, R. Feakins, S. R. Li, S. A. Bustin, and S. Dorudi, "Tumour-infiltrating lymphocytes in colorectal cancer with microsatellite instability are activated and cytotoxic," The British Journal of Surgery, vol. 91, no. 4, pp. 469475, 2004.

[19] E. Elias, D. Mukherji, W. Faraj et al., "Lymph-node ratio is an independent prognostic factor in patients with stage III colorectal cancer: a retrospective study from the Middle East," World Journal of Surgical Oncology, vol. 10, 2012.

[20] W. Ceelen, Y. Van Nieuwenhove, and P. Pattyn, "Prognostic value of the lymph node ratio in stage III colorectal cancer: a systematic review," Annals of Surgical Oncology, vol. 17, no. 11, pp. 2847-2855, 2010.

[21] M. Ferri, L. Lorenzon, M. R. Onelli et al., "Lymph node ratio is a stronger prognotic factor than microsatellite instability in colorectal cancer patients: results from a 7 years follow-up study," International Journal of Surgery, vol. 11, no. 9, pp. 1016-1021, 2013.

[22] A. Emterling, Å. Wallin, G. Arbman, and X. F. Sun, "Clinicopathological significance of microsatellite instability and 
mutated RIZ in colorectal cancer," Annals of Oncology, vol. 15, no. 2, pp. 242-246, 2004.

[23] J. R. Jass, K. A. Do, L. A. Simms et al., "Morphology of sporadic colorectal cancer with DNA replication errors," Gut, vol. 42, no. 5, pp. 673-679, 1998.

[24] J. M. Weiss, P. R. Pfau, E. S. O'Connor et al., "Mortality by stage for right- versus left-sided colon cancer: analysis of surveillance, epidemiology, and end results-medicare data," Journal of Clinical Oncology, vol. 29, no. 33, pp. 4401-4409, 2011.

[25] G. Christodoulidis, M. Spyridakis, D. Symeonidis, K. Kapatou, A. Manolakis, and K. Tepetes, "Clinicopathological differences between right- and left-sided colonic tumors and impact upon survival," Techniques in Coloproctology, vol. 14, Supplement 1, pp. 45-47, 2010.

[26] S. Kang, Y. Na, S. Y. Joung, S. Il Lee, S. C. Oh, and B. W. Min, "The significance of microsatellite instability in colorectal cancer after controlling for clinicopathological factors," Medicine, vol. 97, article e0019, 2018.

[27] K. Öhrling, D. Edler, M. Hallström, and P. Ragnhammar, "Mismatch repair protein expression is an independent prognostic factor in sporadic colorectal cancer," Acta Oncologica, vol. 49, no. 6, pp. 797-804, 2010.

[28] B. A. Magaji, F. M. Moy, A. C. Roslani, and C. W. Law, "Survival rates and predictors of survival among colorectal cancer patients in a Malaysian tertiary hospital," BMC Cancer, vol. 17, article 339, 2017

[29] M. S. Al-Ahwal, Y. H. Shafik, and H. M. Al-Ahwal, "First national survival data for colorectal cancer among Saudis between 1994 and 2004: what's next?" BMC Public Health, vol. 13, no. 1, p. 1, 2013.

[30] M. Radzi, A. Hassan, M. Azri et al., "Survival analysis and prognostic factors for colorectal cancer patients in Malaysia," Annals of Oncology, vol. 17, pp. 3575-3581, 2016.

[31] M. Zhang, Q. C. Zhao, Y. P. Liu, L. Yang, H. M. Zhu, and J. K. Chhetri, "Prognostic analysis and comparison of colon cancer in Han and Hui patients," World Journal of Gastroenterology, vol. 20, no. 17, pp. 5082-5086, 2014. 


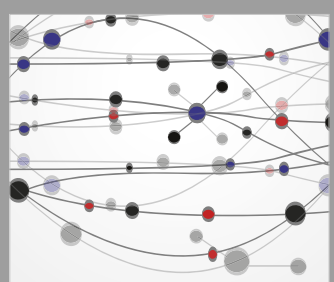

The Scientific World Journal
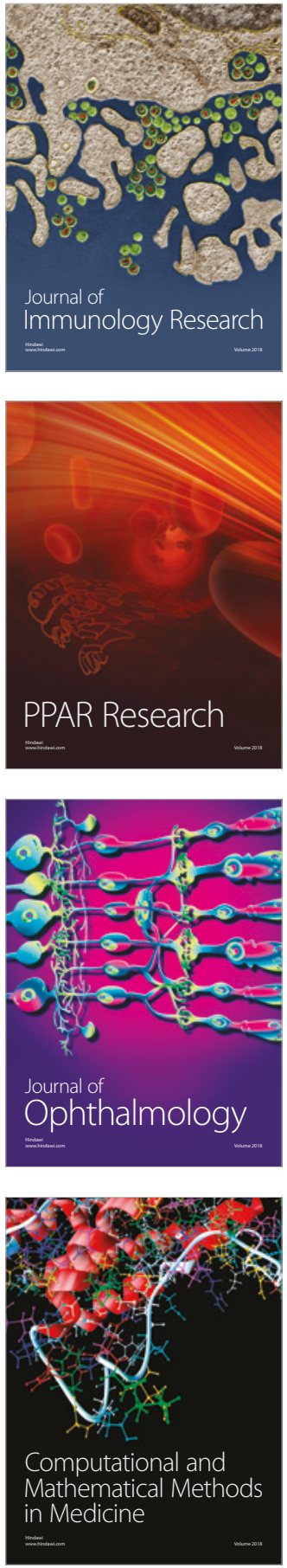

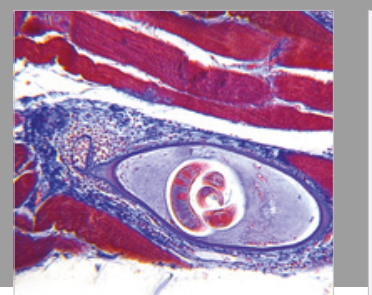

Gastroenterology Research and Practice

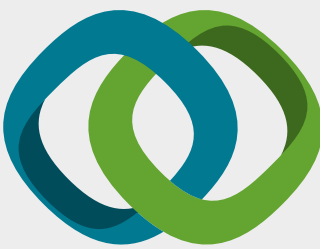

\section{Hindawi}

Submit your manuscripts at

www.hindawi.com
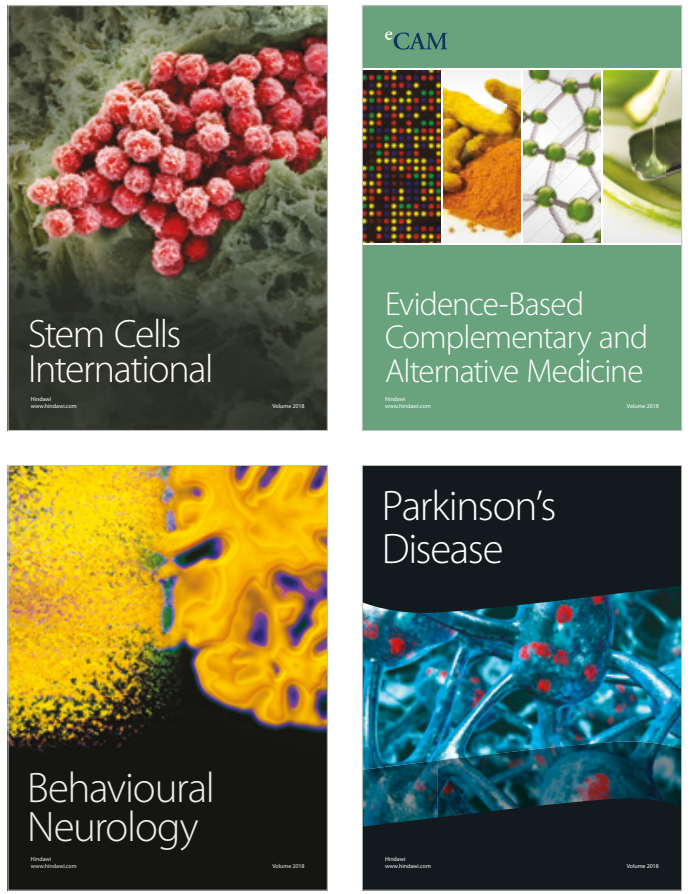

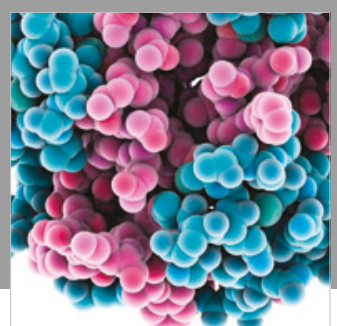

ournal of

Diabetes Research

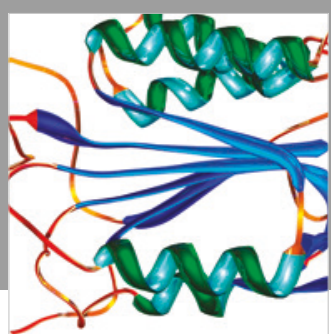

Disease Markers
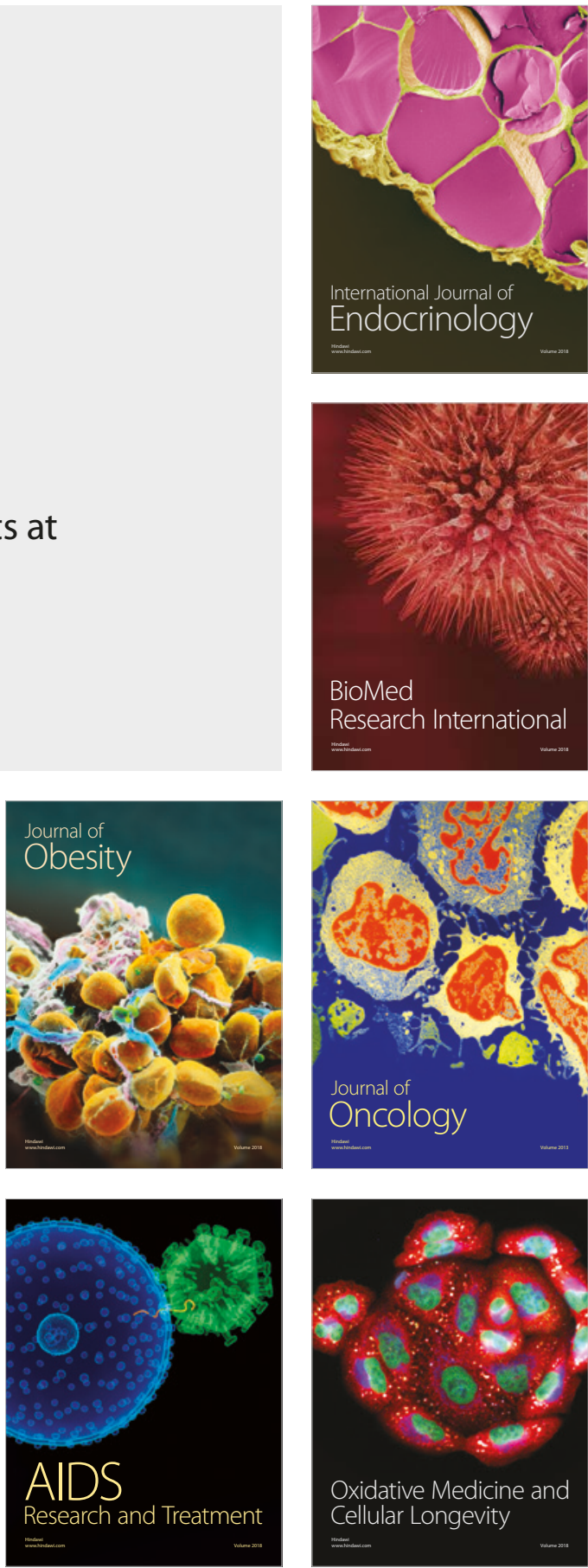